\title{
Importance of operator training and rest perfusion on the diagnostic accuracy of stress perfusion cardiovascular magnetic resonance
}

Adriana D. M. Villa ${ }^{1+}$, Laura Corsinovi ${ }^{1,2+}$, loannis Ntalas ${ }^{1,3}$, Xenios Milidonis $^{1}$, Cian Scannell ${ }^{1}$, Gabriella Di Giovine ${ }^{1}$, Nicholas Child ${ }^{1}$, Catarina Ferreira ${ }^{4}$, Muhummad Sohaib Nazir ${ }^{1}$, Julia Karady ${ }^{1}$, Esmeralda Eshja ${ }^{5}$, Viola De Francesco ${ }^{1}$, Nuno Bettencourt ${ }^{6}$, Andreas Schuster ${ }^{7,8,9}$, Tevfik F. Ismail ${ }^{1}$, Reza Razavi ${ }^{1}$ and Amedeo Chiribiri ${ }^{1 *}$

\begin{abstract}
Background: Clinical evaluation of stress perfusion cardiovascular magnetic resonance (CMR) is currently based on visual assessment and has shown high diagnostic accuracy in previous clinical trials, when performed by expert readers or core laboratories. However, these results may not be generalizable to clinical practice, particularly when less experienced readers are concerned. Other factors, such as the level of training, the extent of ischemia, and image quality could affect the diagnostic accuracy. Moreover, the role of rest images has not been clarified.

The aim of this study was to assess the diagnostic accuracy of visual assessment for operators with different levels of training and the additional value of rest perfusion imaging, and to compare visual assessment and automated quantitative analysis in the assessment of coronary artery disease (CAD).

Methods: We evaluated 53 patients with known or suspected CAD referred for stress-perfusion CMR. Nine operators (equally divided in 3 levels of competency) blindly reviewed each case twice with a 2-week interval, in a randomised order, with and without rest images. Semi-automated Fermi deconvolution was used for quantitative analysis and estimation of myocardial perfusion reserve as the ratio of stress to rest perfusion estimates.

Results: Level-3 operators correctly identified significant CAD in $83.6 \%$ of the cases. This percentage dropped to $65.7 \%$ for Level-2 operators and to $55.7 \%$ for Level-1 operators $(p<0.001)$. Quantitative analysis correctly identified CAD in $86.3 \%$ of the cases and was non-inferior to expert readers $(p=0.56)$. When rest images were available, a significantly higher level of confidence was reported $(p=0.022)$, but no significant differences in diagnostic accuracy were measured $(p=0.34)$.

Conclusions: Our study demonstrates that the level of training is the main determinant of the diagnostic accuracy in the identification of CAD. Level-3 operators performed at levels comparable with the results from clinical trials. Rest images did not significantly improve diagnostic accuracy, but contributed to higher confidence in the results. Automated quantitative analysis performed similarly to level-3 operators. This is of increasing relevance as recent technical advances in image reconstruction and analysis techniques are likely to permit the clinical translation of robust and fully automated quantitative analysis into routine clinical practice.
\end{abstract}

Keywords: Cardiovascular magnetic resonance, Stress perfusion imaging, Coronary artery disease, Quantitative assessment, Myocardial ischemia, Diagnostic accuracy, Training

\footnotetext{
* Correspondence: amedeo.chiribiri@kcl.ac.uk

${ }^{\dagger}$ Adriana D. M. Villa and Laura Corsinovi contributed equally to this work.

'School of Biomedical Engineering \& Imaging Sciences, King's College

London, King's Health Partners, 4th Floor Lambeth Wing, St Thomas'

Hospital, London SE1 7EH, UK

Full list of author information is available at the end of the article
}

(c) The Author(s). 2018 Open Access This article is distributed under the terms of the Creative Commons Attribution 4.0 International License (http://creativecommons.org/licenses/by/4.0/), which permits unrestricted use, distribution, and reproduction in any medium, provided you give appropriate credit to the original author(s) and the source, provide a link to the Creative Commons license, and indicate if changes were made. The Creative Commons Public Domain Dedication waiver (http://creativecommons.org/publicdomain/zero/1.0/) applies to the data made available in this article, unless otherwise stated. 


\section{Background}

Stress perfusion cardiovascular magnetic resonance (CMR) is increasingly used for the evaluation of patients with known or suspected coronary artery disease (CAD) and has a class I indication for patients at intermediate risk of CAD according to recent guidelines [1, 2].

Stress perfusion CMR has been shown to be highly accurate for the detection of CAD, with sensitivity ranging from 75 to $91 \%$ and specificity ranging from 59 to $87 \%$ [3-5]. It should be noted that in most of these studies, visual assessment has been carried out either by a core laboratory or by expert readers, and therefore the findings may not be generalizable to routine clinical practice. As stress perfusion CMR gains acceptance and becomes more available, it will inevitably be performed in lower volume and less experienced centers.

Stress perfusion CMR is typically evaluated by visual assessment. This can be influenced by the extent of ischemia and the presence of areas of relatively preserved perfusion, which can be used as reference [6]. Moreover, image artefacts can complicate the interpretation of the images. Dark rim artefacts, which are commonly observed during stress perfusion, can be misdiagnosed as subendocardial perfusion abnormalities [7], in particular when relatively long acquisition times are used and spatial resolution is low. Moreover, areas of infarction are frequently associated with delayed perfusion $[8,9]$. The simultaneous evaluation of stress and rest perfusion $\mathrm{CMR}$ and late gadolinium enhancement (LGE) images is recommended to identify areas of myocardial infarction and improve the specificity of the interpretation $[10,11]$, and to exclude imaging artefacts [10].

Additionally, it has been suggested that rest perfusion images could play an important role in improving the identification of imaging artefacts when signal abnormalities are present on both stress and rest images [10]. The acquisition of rest images enables quantification of perfusion reserve, but prolongs scan times and requires additional contrast dosing.

Stress perfusion CMR is complex to read and requires significant training and experience. However, the impact of training and experience has not been formally studied and as yet, there are no specific recommendations in current guidelines, apart from stating that stress perfusion CMR should be part of the training program for Level-2 readers [12]. It is hoped that fully quantitative automated methods may help bridge training gaps and support clinical decision making.

We sought to determine the importance of the level of training of the operator on the diagnostic accuracy of stress perfusion CMR; the role of rest perfusion images in the identification of imaging artefacts and in the correct detection of CAD; and to systematically compare the results of visual assessment with semi-automated quantitative analysis to determine its additional value.

\section{Methods}

Consecutive patients $(n=53)$ referred for stress perfusion CMR for suspected CAD were retrospectively included in the study. All patients had invasive coronary angiography on the basis of the clinical indication within 1 month of the CMR examination. Exclusion criteria were contraindications to CMR, gadolinium-based contrast agents or adenosine. Patients with previous coronary artery bypass grafting, hypertrophic cardiomyopathy, aortic stenosis, or other primary myopathic or valvular disease were excluded. All subjects gave written informed consent in accordance with ethical approval. This study complies with the Declaration of Helsinki.

\section{Image acquisition}

CMR images were acquired using a 3T scanner (Achieva, Philips Healthcare, Best, The Netherlands) equipped with 32-channel phased-array cardiac coil. The protocol included functional assessment, adenosine stress and rest first pass perfusion imaging, and LGE. The images were acquired using standard acquisition protocols and in end-expiratory breath-hold. For stress imaging, $140 \mu \mathrm{g} /$ $\mathrm{kg} / \mathrm{min}$ of adenosine was administered. Imaging commenced at least 3 minutes after infusion initiation. A dual bolus (equal volumes of $0.0075 \mathrm{mmol} / \mathrm{kg}$ followed by $0.075 \mathrm{mmol} / \mathrm{kg}$ after a 20-s pause) of contrast agent (gadobutrol/Gadovist, Schering, Germany) was injected at $4 \mathrm{ml} /$ $\mathrm{s}$ by a power injector [13]. For perfusion, a saturation recovery prepared gradient echo pulse sequence accelerated with $\mathrm{k}-\mathrm{t}$ sensitivity encoding acceleration with 11 training profiles was used. Typical imaging parameters were: 3 short-axis slices covering standard American Heart Association (AHA) segments [14], 120 acquired dynamics/ slice, flip angle $20^{\circ}$, TR $2.5 \mathrm{~ms}$, TE $1.25 \mathrm{~ms}$, saturation pre-pulse recovery time $100 \mathrm{~ms}$, pixel size $1.9 \times 1.9 \mathrm{~mm}$, slice thickness $10 \mathrm{~mm}$.

Typical imaging parameters for LGE imaging were: long and short axis to fully cover the left ventricle, inversion recovery turbo field echo, flip angle $25^{\circ}$, TR $6 \mathrm{~ms}$, TE $3 \mathrm{~ms}$, pixel size $0.7 \times 0.7 \mathrm{~mm}$, slice thickness $10 \mathrm{~mm}$.

\section{Operator selection}

Nine operators were chosen amongst the physicians working in our unit and in other European institutions, on the basis of their level of competency, according to the European Society of Cardiology (ESC)/European Association of Cardiovascular Imaging (EACVI) training guidelines [12]. A total of 9 operators, 3 for each competency level, were chosen; all operators had recently obtained the ESC/EACVI certification (within 2 months) for the appropriate level. In brief, level-1 competency ESC certification requires 20 continuous medical education (CME) hours, involvement in $50 \mathrm{CMR}$ cases and 1-month fellowship; level-2 requires at least $50 \mathrm{CME}$ 
hours, involvement in 150 clinical cases of which 25 must be perfusion studies, a minimum of 3-months fellowship and the European CMR exam; level-3 requires at least $50 \mathrm{CME}$ hours, involvement in 300 clinical cases of which a minimum of 50 must be perfusion studies, at least 12-months training and the European CMR exam. Level-1 competency reflects core CMR training, level-2 is required to report CMR studies with support from a Level-3 operator and Level-3 is required to perform, interpret and report CMR studies fully independently [12].

\section{Image analysis - Visual assessment}

Each operator was asked to report each of the 53 scans twice over a 4-week period, with a minimum interval of 2 weeks between first and second read. The scans were anonymized and presented to the operator as a full dataset, including stress and rest perfusion and LGE, or as reduced datasets, including stress perfusion and LGE only. The full and reduced datasets were analysed blinded to clinical and angiographic data and in a randomized order on different days. The study flowchart can be seen in Fig. 1 .

Visual assessment of adenosine stress perfusion CMR and LGE images, displayed side-by-side, was performed as per clinical practice, in accordance with standardized CMR protocols [15]. A perfusion defect was defined as a regional reduction in myocardial signal during LV first-pass of contrast agent, not related to artefacts and not corresponding to an area of scar on LGE images.

Operators were asked to fill an on-line standardized form and to identify segments with inducible ischemia, to identify the presence and transmurality of LGE [16], to identify the most likely culprit coronary artery based on the standard AHA segmentation [14], and to grade their confidence in the diagnosis and the perceived image quality.
The confidence was graded as: 0- very unconfident, 1unconfident, 2- confident, 3- very confident. The perceived image quality was graded as: 0 - poor, 1 - moderate, 2- good, 3- excellent.

Coronary angiography results have been used as reference standard. The threshold for coronary artery lumen stenosis was 70\% diameter stenosis for epicardial vessels. All invasive angiographic images have been reviewed by consensus of expert operators.

\section{Image analysis - Quantitative assessment}

A different operator, blinded to results of visual perfusion assessment and other clinical/angiographic data, performed the segmentation of the images for semi-automated quantitative analysis using software and methods previously developed and validated by our group. Respiratory motion was corrected using affine image registration by maximization of the joint correlation between consecutive dynamics within an automatically determined region of interest [17]. A temporal maximum intensity projection was calculated to serve as a feature image for automatic contour delineation method. The operator then manually optimized the automatically generated contours to avoid partial volume effects at the endocardial and epicardial borders [17]. The intervention of the operator was limited to image segmentation. Quantitative perfusion analysis was then automatically performed by Fermi-constrained deconvolution according to the methods described by Wilke et al. [18] and Jerosch-Herold et al. [19], optimised for high-resolution pixel-wise analysis [20, 21]. Myocardial perfusion reserve (MPR) was calculated as the ratio between stress and rest myocardial blood flow (MBF) estimates. Ischemia was defined as segments with MPR $<1.5$, according to previously validated criteria $[22,23]$.

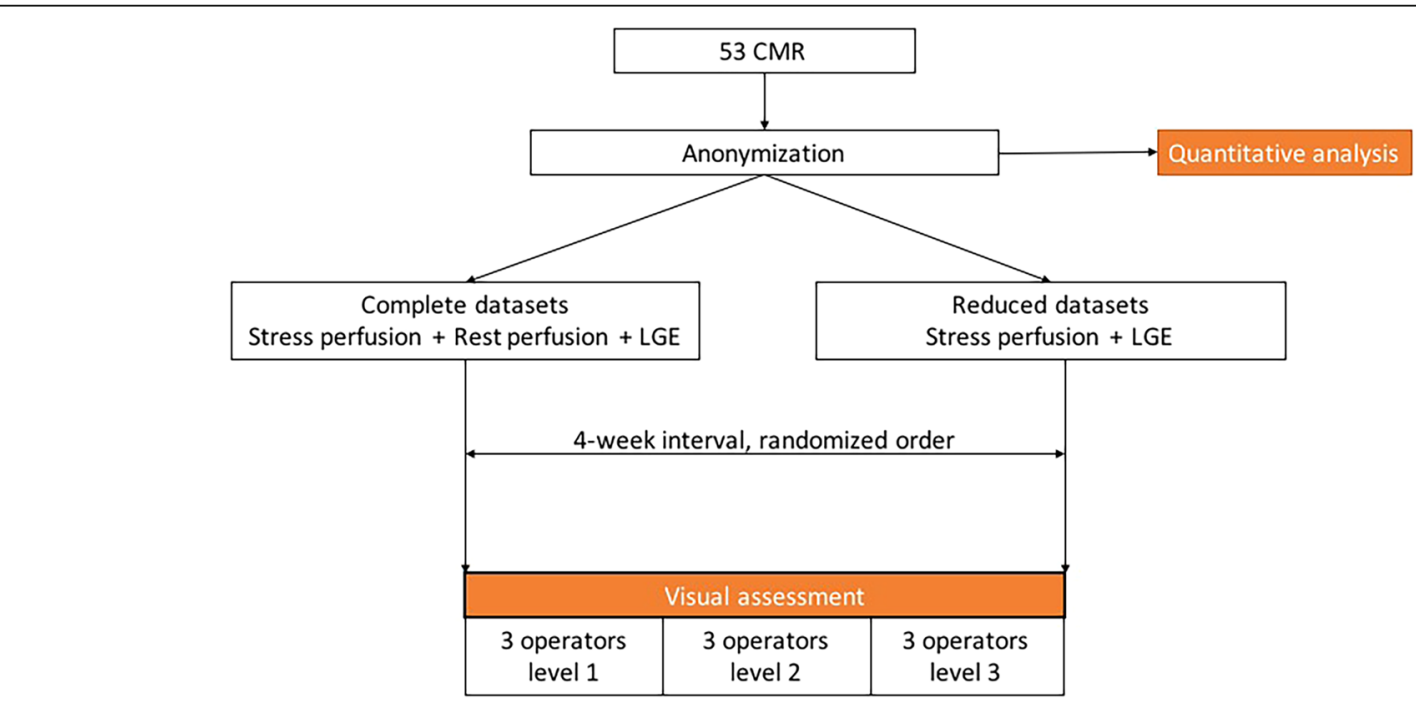

Fig. 1 Study flowchart. CMR: cardiovascular magnetic resonance, LGE: late gadolinium enhancement 


\section{Statistical analysis}

Continuous variables are presented as mean \pm standard deviation for normally distributed variables and as median with interquartile range for non-parametric data. Normality was assessed with Q-Q plots and the Kolmogorov-Smirnov test. Continuous variables were compared using an unpaired Student $t$ test or the Wilcoxon rank-sum test, as appropriate, and categorical data were compared between groups using the Fisher exact test and Pearson chi-square test. The McNemar test was used for paired dichotomous data. Two-tailed values of $p<0.05$ were considered to be statistically significant. One-way ANOVA was used to determine differences between multiple groups. Bonferroni correction was used to account for multiple testing.

\section{Results}

\section{Characteristics of the population}

The mean age of the population $(n=53)$ was $60.6 \pm$ 12.7 years. Demographic data are shown in Table 1. The prevalence of CAD in the group of patients included in the analysis was $30.2 \%$, with $16 / 53$ patients positive for CAD on invasive coronary angiography. Left anterior descending (LAD) lesions were identified in 9 (17\%) of the cases, left circumflex (LCX) lesions in 8 (15.1\%) of the cases, and right coronary artery (RCA) in 13 (24.5\%) of the cases. Within the group of patients with CAD, 8 patients had 1-vessel disease (50\%), 5 patients 2 -vessel disease (31.3\%) and 3 patients 3 -vessel disease (18.8\%).

\section{Impact of operator training on correct CAD identification}

There was a significant correlation between an operator's training level and the rate of correct identification of $\mathrm{CAD}$ on a per patient level on visual assessment. The diagnosis of Level-3 operators agreed with invasive coronary angiography in $83.6 \pm 2.3 \%$ of the cases, while this percentage dropped to $65.7 \pm 4.3 \%$ for Level- 2 operators and to $55.7 \pm 5.3 \%$ for Level- 1 operators $(p<0.001$ between the 3 groups) (Fig. 2). A significant difference in the agreement with angiography between different levels of training was also observed in a sub-analysis per

Table 1 Demographic characteristics of the population

\begin{tabular}{ll}
\hline & All $(n=53)$ \\
\hline Age (years) & $60.6 \pm 12.7$ \\
Male gender & $36(67.9 \%)$ \\
Hypertension & $30(56.6 \%)$ \\
Dyslipidaemia & $23(43.4 \%)$ \\
Diabetes & $10(18.9 \%)$ \\
Current smoker & $13(24.5 \%)$ \\
Previous PCl & $9(17 \%)$ \\
Family history of CAD & $12(22.6 \%)$ \\
\hline PCl percutaneous coronary artery intervention, CAD coronary artery disease
\end{tabular}

coronary territory $(\mathrm{p}<0.001)$ (Fig. 3). When different perfusion territories were compared, the agreement between CMR and coronary angiography was higher for the LAD territory, followed by the LCX and by the RCA territories. The same trend was observed in all groups of operators, regardless of the level of training $(\mathrm{p}<0.001)$.

The sensitivity and specificity for operators of different levels of training are reported in Fig. 4. Level-1 operators showed high sensitivity $(86.5 \pm 6.1 \%)$ and low specificity $(41.9 \pm 10.9 \%)$. Level-2 operators had a sensitivity of $57.3 \pm 4.7 \%$ and a specificity of $69.4 \pm 9.9 \%$. Level- 3 operators showed a sensitivity of $71.9 \pm 13 \%$ and a specificity of $88.7 \pm 6.7 \%$ respectively. There was a statistically significant difference for both sensitivity and specificity between different levels of training $(\mathrm{p}<0.001)$ (Fig. 4).

\section{Impact of rest perfusion on correct identification of CAD}

When rest images were available, there was no statistically significant difference at all levels of training (Fig. 5) and in the overall analysis $(69.6 \pm 14.3 \%$ vs $67.1 \pm 13.1 \%$; $p=0.34$ ). However, when rest images were available, a significantly higher level of confidence was reported by the operators $(p=0.022)$ and subjective image quality was scored at a higher level $(p=0.012)$.

\section{CAD classification}

Figure 6 shows a comparison between the extent of CAD identified by the operators on CMR images in comparison with invasive coronary angiography. An overestimation of the severity of CAD was observed in Level-1 operators, regardless of the number of vessels with CAD. Despite being more accurate, Level-2 and Level-3 operators significantly underestimated the number of positive perfusion territories in patients with multi-vessel CAD.

\section{Impact of quantitative analysis on correct CAD identification}

Quantitative analysis was successfully performed in 51 patients. In 2 cases of patients without CAD, the automated algorithms failed and no results could be calculated. In both cases, this was due to the low quality of the diluted pre-bolus used for the estimation of the arterial input function. Level-3 visual assessment of the 2 cases where quantification failed yielded the correct diagnosis in both cases when both stress and rest images were made available to the readers, and in $66 \%$ of interpretations when only stress perfusion was made available to the readers. Quantitative stress perfusion CMR analysis agreed with the results of invasive angiography in $86.3 \%$ of the cases, performing significantly better than Level-1 and Level-2 operators $(p<0.001)$. Level-3 visual assessment and quantitative analysis were not significantly different ( $p=0.56)$ (Fig. 2). Quantitative analysis had a sensitivity of $68.8 \%$ and specificity of $94.3 \%$. When 


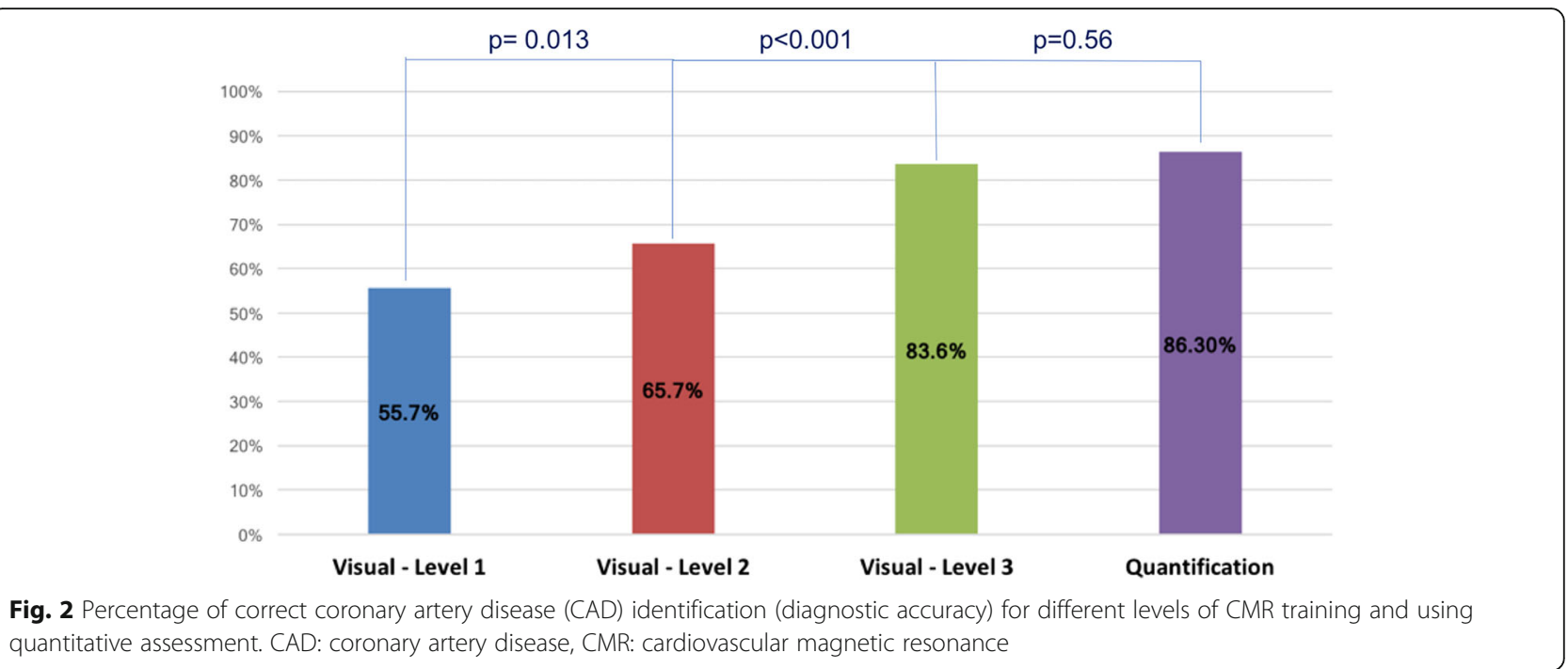

the 2 cases in which quantitative analysis failed are considered as a missed diagnosis, the concordance of quantitative analysis with invasive angiography was $83 \%$, with a sensitivity of $68.8 \%$ and a specificity of $89.2 \%$.

\section{Discussion}

This study has several important findings. Operator training and experience had a significant impact on diagnostic accuracy. Only Level-3 trained operators had an accuracy comparable with the results reported by large clinical trials [3-5]. Rest images did not significantly improve the diagnostic accuracy of stress perfusion CMR but, when available, contributed to a significantly higher confidence of the operators in their reports and to a higher perceived image quality, regardless of the level of training. Finally, semi-automated quantitative analysis performed better than Level-1 and Level-2 operators, but similarly to a Level-3 operator. Quantitative analysis however failed in 2/53 cases due to technical reasons related to the administration of the diluted pre-bolus. However, the same cases could be analysed visually.

Stress perfusion CMR plays an increasingly important role in the evaluation of patients with known or suspected CAD. Recent European guidelines recommend the use of stress perfusion CMR in patients with suspected CAD and intermediate pre-test probability, with a class 1 indication and level of evidence A, similarly to stress echocardiography and nuclear imaging $[1,2]$. US guidelines recommend stress perfusion CMR with 2A indication [24], particularly in specific subgroups of patients [25]. These indications are based on the assumption that stress perfusion CMR is highly accurate for the identification of CAD and compares favorably with other functional modalities. In large trials and meta-analyses, the sensitivity ranged

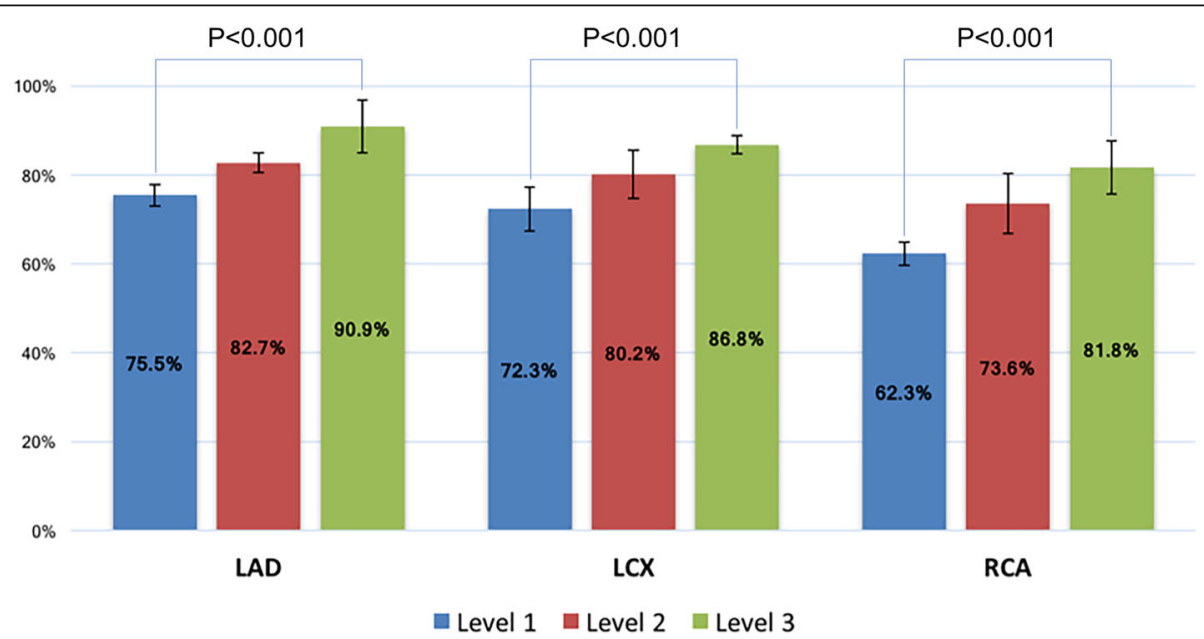

Fig. 3 Percentage of correct CAD identification (diagnostic accuracy) stratified by coronary territory. CAD: coronary artery disease, LAD: left anterior descending coronary artery, LCX: left circumflex coronary artery, RCA: right coronary artery 


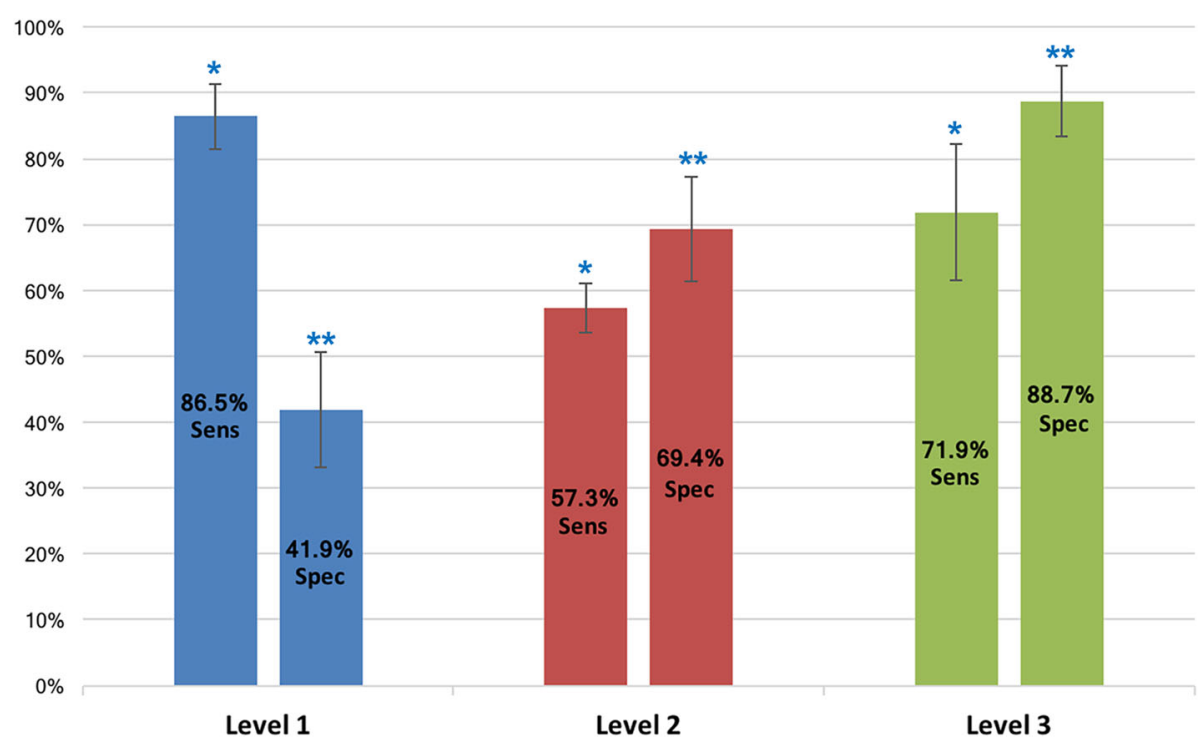

Fig. 4 Sensitivity and specificity for level of CMR training. * denotes statistically significant difference $(p<0.001)$ between sensitivity values. ** denotes statistically significant difference $(p<0.001)$ between specificity values. Sens: sensitivity, spec: specificity

from $75 \%$ [3] to $91 \%$ [4] and specificity ranged from 59\% [3] to $87 \%$ [5]. In the CE-MARC study [26], sensitivity was $86.5 \%$ and specificity was $83.4 \%$, and the MR-IMPACT 2 trial [27] reported a sensitivity of $75 \%$ and a specificity of $59 \%$. These wide intervals most likely represent the variability in study design, the different prevalence of disease in different populations, and variability in the criteria used for visual assessment.

The diagnostic accuracy of stress perfusion CMR reported in the literature is often the result of visual assessment carried out by expert readers, which are usually Level-3 operators and often are internationally recognized experts.
Our study demonstrates that the diagnostic accuracy varied significantly amongst groups of readers with different levels of training, and reached values comparable with those of large studies only in the group of Level-3 operators. These results confirm the high diagnostic accuracy of stress perfusion CMR in comparison with coronary angiography, however clearly indicate the need for Level-3 supervision when stress perfusion scans are reported.

From the analysis of the sensitivity and specificity for the detection of CAD in different groups, it emerges that Level-1 operators had high sensitivity (86.5\%). This came however at the cost of a reduced specificity $(41.9 \%)$ and rate of overall correct CAD detection (55.7\%). Factors such as

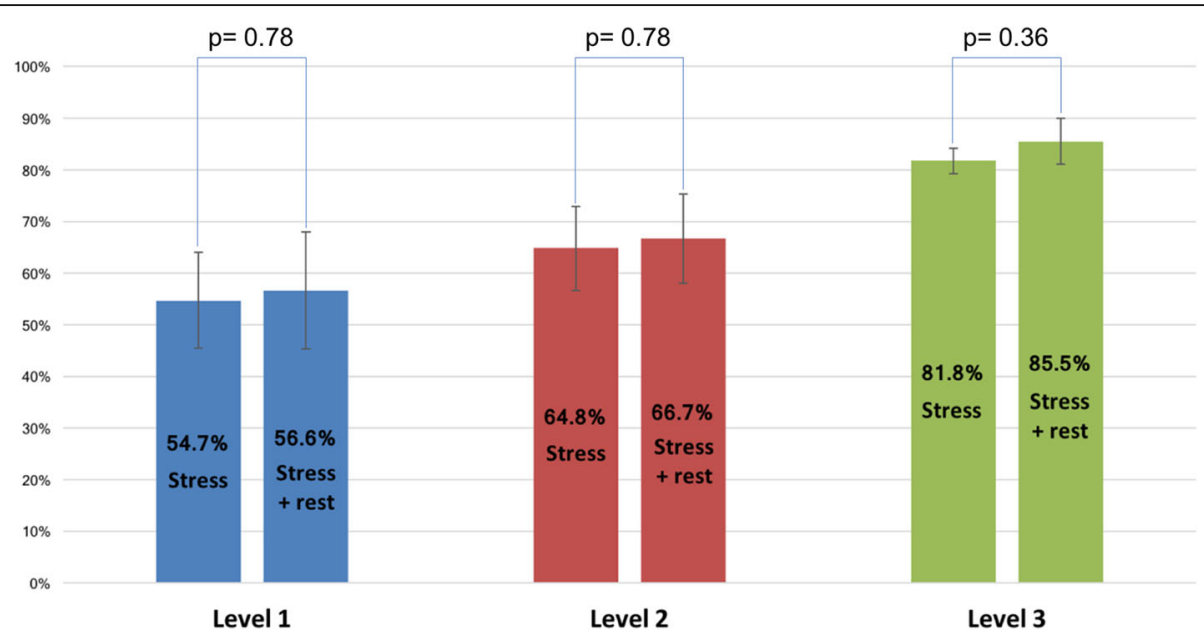

Fig. 5 Percentage of correct identification of CAD (diagnostic accuracy) using stress perfusion only or stress and rest images. CAD: coronary artery disease 


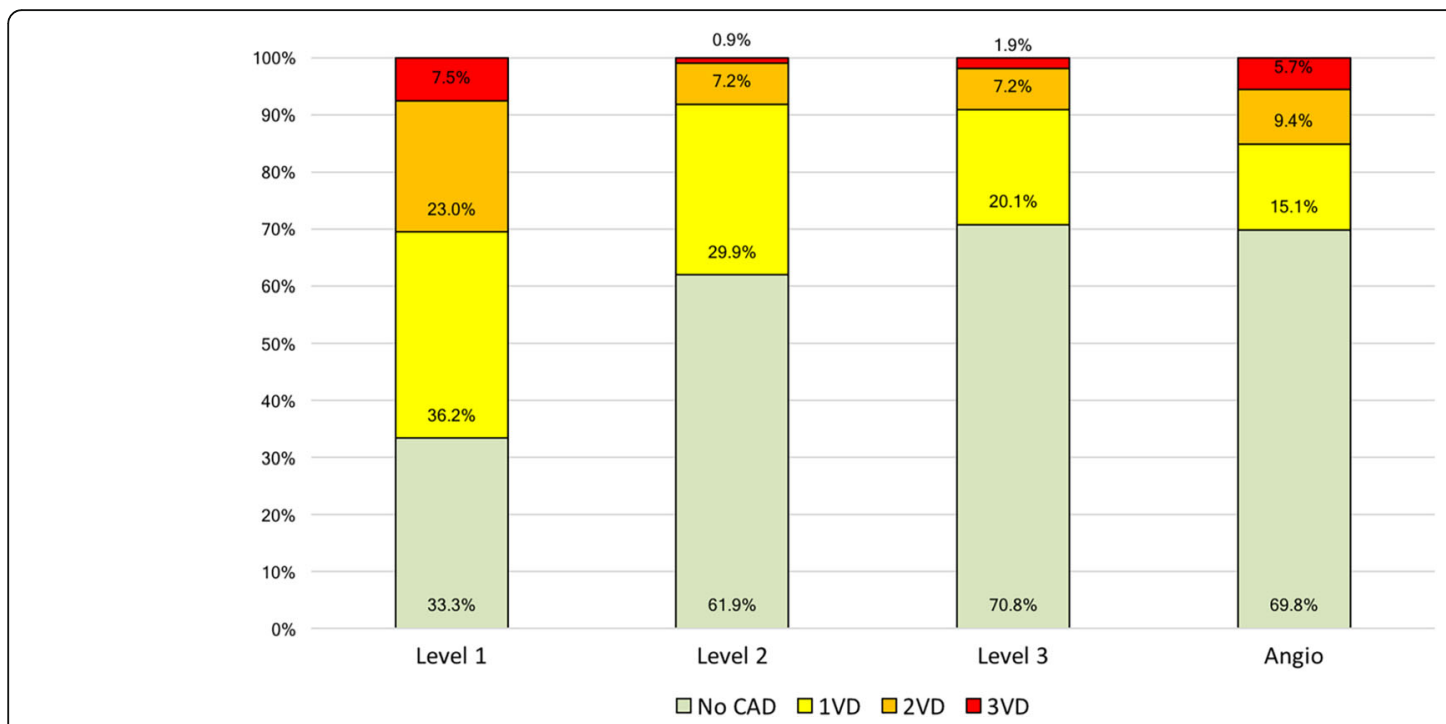

Fig. 6 CAD classification for different levels of CMR training. CAD: coronary artery disease, 1VD: one-vessel disease, 2VD, two-vessel disease, 3VD: three-vessel disease

image quality and the prevalence of dark rim artefacts, which can mimic the presence of subendocardial perfusion defects, could have played a role. In comparison, Level-3 operators under-called the disease (sensitivity $71.9 \%$ ), but had a high specificity (88.7\%). All diagnostic investigations involve a trade-off between sensitivity and specificity. At a population level and from a health-economic perspective, we feel that the results achieved by Level 3 operators represent a reasonable balance between the need to identify significant coronary disease and the high specificity required to avoid increasing down-stream investigation costs through increased referral for invasive coronary angiography. The work of Patel et al. [28] highlights the need for better selection of patients for invasive investigation given the costs and potential morbidity incurred by this.

Our results support the recommendations from the ESC [12], which state that Level-1 operators hold the basic knowledge in CMR sufficient to select appropriate CMR indications and interpret CMR reports, but are not cleared to report CMR scans. This is reflected in our result by the fact that Level-1 operators demonstrated a very low diagnostic accuracy, with poor specificity for the presence of CAD. According to the ESC guidelines, Level-2 operators may actively perform and report CMR, but are not completely independent and should work under the supervision of a Level-3 expert. This is also supported by our results, since Level-2 operators were significantly less accurate than Level-3 operators. Level-3 operators instead performed to levels similar to those reported by studies such as the CE-MARC [26].

It should be noted that the Society for Cardiovascular Magnetic Resonance (SCMR) guidelines on training [29] differ slightly from the ESC guidelines used in this study to define the level of training of the operators. According to the SCMR guidelines, Level-2 operators can independently report CMR scans, whereas Level- 3 certification has more to do with being able to lead a CMR unit and perform research in the field. Both guidelines agree that Level-1 training is not sufficient to practice CMR.

It has been suggested that rest perfusion images play an important role in improving the identification of imaging artefacts when signal abnormalities are present on both stress and rest images [10]. When assessing stress perfusion CMR visually, guidelines advise displaying both rest and stress images side-by-side to identify correctly inducible perfusion defect and artefacts $[10,11]$.

In our study, we did not find any significant difference in the diagnostic accuracy when rest images were available. Our findings mirror those of Biglands et al. [30]. However, when testing the operator confidence and the perceived image quality, a statistically significant difference was noted when both stress and rest images were available. The increased confidence was more evident for Level-1 and Level-2 operators.

Interestingly, Level-1 operators reported a higher confidence score than more experienced operators, despite lower overall accuracy. This could reflect a cognitive bias, also known as the Dunning-Kruger effect [31].

The diagnostic usefulness of rest perfusion imaging resides in the finding of "fixed perfusion defect" on both stress and rest images, which may be related to artefacts or to areas of myocardial infarction. However, this may be overcome when stress perfusion CMR is assessed visually side-by-side with LGE, as per guidelines [11] and as in our study. Nevertheless, rest perfusion imaging remains a fundamental requirement for perfusion quantification and MPR estimation. 
Semi-automated quantitative assessment performed better than Level-1 and Level-2 operators and similarly to Level-3 operators for the detection of CAD. The latter is in keeping with the results of several other studies that reported high sensitivity and specificity for quantitative analysis, with sensitivity ranging from $80 \%$ [22] to $94.4 \%$ [32] and specificity ranging from $81 \%$ [33] to $100 \%$ [34]. Previous studies from Patel et al. [6] and Mordini et al. [35] compared quantitative with visual and semi-quantitative analysis and demonstrated that quantitative analysis is superior to visual assessment and semi-quantitative assessment in the detection of ischemia, and that quantitative analysis is the most accurate method to measure the total ischemic burden.

In the present study, quantitative analysis was performed using a semi-automated method which requires user input to confirm the automated segmentation of the images, but eliminates inter-observer variability for what concerns the quantification procedure. This is of increasing relevance as recent technical advances in image reconstruction and analysis techniques are likely to permit the clinical translation of robust and fully automated quantitative analysis into routine clinical practice [36-39]. In our study however, the dual bolus approach used for arterial input function measurements failed in 2 subjects, impeding quantitative analysis. The advent of dual sequences capable of a more accurate assessment of the concentration of gadolinium in the main bolus input function may make the use of dual bolus redundant in the near future $[37,40]$.

\section{Limitations}

This study included a selected population with suspected CAD and we excluded patients with primary cardiomyopathy. Thus, our results on diagnostic accuracy do not include other patterns of perfusion abnormalities, which may require even more experience to discern (e.g., microvascular dysfunction).

Moreover, we used an anatomical reference standard (invasive coronary angiography) to compare operators' performances in interpreting a functional test, while a functional reference standard (e.g., fractional flow reserve) may be more appropriate.

Our results demonstrate that similarly accurate detection of CAD can be achieved by Level-3 operators and by automated perfusion quantification. Although our study was not powered to demonstrate the superiority of quantitative analysis, this has been the subject of a recent study which has reported very similar findings [30]. The non-inferiority of automated quantification to expert visual reads, in combination with the prognostic value of quantitative analysis [23] will facilitate more widespread adoption of stress perfusion CMR by less experienced readers.
Finally, all stress perfusion CMR were acquired in a single center, using a $3 \mathrm{~T}$ Philips scanner and a high-resolution $\mathrm{k}-\mathrm{t}$ sequence. This may not reflect the standard clinical acquisition in other centres.

\section{Conclusions}

This study demonstrates that visual assessment of stress perfusion CMR is challenging for Level-1 and Level-2 operators but accurate in the hands of Level-3 operators. Our results highlight the importance of the recommendations of the ESC/EACVI training guidelines in CMR, which recommend independent reporting for Level-3 operators only and supervised reporting for Level-2 trained operators. The availability of rest perfusion images was associated with significantly higher confidence and higher perceived image quality, regardless of the level of training of the operator. Quantitative analysis performed similarly to Level-3 trained operators and could represent, in the future, a valid alternative to visual assessment.

\section{Abbreviations \\ AHA: American Heart Association; CAD: Coronary artery disease; \\ CME: Continuous medical education; CMR: Cardiovascular magnetic resonance; EACVl: European Association of Cardiovascular Imaging; ESC: European Society of Cardiology; LAD: Left anterior descending coronary artery; LCX: Left circumflex coronary artery; LGE: Late gadolinium enhancement: MBF: Myocardial blood flow; MPR: Myocardial perfusion reserve; RCA: Right coronary artery; SCMR: Society for Cardiovascular Magnetic Resonance}

\section{Acknowledgements}

This work was supported by the National Institute for Health Research (NIHR) Cardiovascular Health Technology Cooperative (HTC) and Biomedical Research Centre (BRC) awarded to Guy's \& St Thomas' NHS Foundation Trust in partnership with King's College London. The views expressed are those of the author(s) and not necessarily those of the NHS, the NIHR or the Department of Health. This work was additionally supported by the Wellcome/EPSRC Centre for Medical Engineering at King's College London [WT 203148/Z/16/Z].

We are grateful to the radiographers at the School of Biomedical Engineering and Imaging Sciences at King's College London for their support in the acquisition of the images.

MSN was funded through a fellowship from the Medical Research Council [MR/P01979X/1].

\section{Funding}

This work was supported by the National Institute for Health Research (NIHR) Cardiovascular Health Technology Cooperative (HTC) and Biomedical

Research Centre (BRC) awarded to Guy's \& St Thomas' NHS Foundation Trust in partnership with King's College London. This work was additionally supported by the Wellcome/EPSRC Centre for Medical Engineering at King's College London [WT 203148/Z/16/Z]. MSN was funded through a fellowship from the Medical Research Council [MR/P01979X/1]. All funding bodies equally contributed to the collection, analysis, and interpretation of the data and to the investigators' salaries during the study.

\section{Availability of data and materials}

Please contact author for data requests.

\section{Authors' contributions}

ADMV, LC, NB and AC conceived the study and participated in the study design and coordination. IN, GDG, NC, CF, MSN, JK, ES, VDF and AS analysed the data. ADMV, LC, XM, CS and TFI performed the data analysis. AC segmented the data for quantitative analysis. ADMV and LC drafted the manuscript. All authors critically revised the manuscript for important intellectual content, read and approved the final manuscript. 


\section{Ethics approval and consent to participate}

All subjects gave written informed consent in accordance with ethical approval (ethics 15/NS/0030, NHS Grampians Regional Ethics Committee).

\section{Consent for publication}

Not applicable.

\section{Competing interests}

The authors declare that they have no competing interests.

\section{Publisher's Note}

Springer Nature remains neutral with regard to jurisdictional claims in published maps and institutional affiliations.

\begin{abstract}
Author details
'School of Biomedical Engineering \& Imaging Sciences, King's College London, King's Health Partners, 4th Floor Lambeth Wing, St Thomas' Hospital, London SE1 7EH, UK. ${ }^{2}$ Cardiology Department of the Basingstoke and North Hampshire Hospital, Basingstoke, UK. ${ }^{3}$ Cardiology Department, St. Thomas' Hospital, Guy's and St Thomas' NHS Foundation Trust, London, UK. ${ }^{4}$ Faculdade de Ciências da Saúde CICS, UBI, Covilhã, Portugal. ${ }^{5}$ Radiology Department, ICS Maugeri IRCCS, Pavia, Italy. ${ }^{6}$ Cardiovascular R\&D Unit, Faculty of Medicine, University of Porto, Porto, Portugal. ${ }^{7}$ Department of Cardiology, Royal North Shore Hospital, The Kolling Institute, Northern Clinical School, University of Sydney, Sydney, Australia. ${ }^{8}$ Department of Cardiology and Pneumology, University Medical Center Göttingen, Georg-August University, Göttingen, Germany. ${ }^{9}$ German Center for Cardiovascular Research (DZHK), Partner Site Göttingen, Göttingen, Germany.
\end{abstract}

\section{Received: 1 March 2018 Accepted: 9 October 2018}

\section{Published online: 19 November 2018}

\section{References}

1. Task Force Members, Montalescot G, Sechtem U, Andreotti F, Arden C, Budaj $A$, et al. ESC guidelines on the management of stable coronary artery disease: The Task Force on the management of stable coronary artery disease of the European Society of Cardiology. Eur Heart J. 2013;34:2949-3003.

2. Authors Task Force members, Kolh P, Alfonso F, Collet J-P, Cremer J, Falk V, et al. ESC/EACTS Guidelines on myocardial revascularization. Eur Heart J. 2014;35:ehu278-619 The Oxford University Press.

3. Schwitter J, Wacker CM, Wilke N, Al-Saadi N, Sauer E, Huettle K, et al. Superior diagnostic performance of perfusion-cardiovascular magnetic resonance versus SPECT to detect coronary artery disease: the secondary endpoints of the multicenter multivendor MR-IMPACT II (magnetic resonance imaging for myocardial perfusion assessment in coronary artery disease trial). J Cardiovasc Magn Reson. 2012;14:1.

4. Nandalur KR, Dwamena BA, Choudhri AF, Nandalur MR, Carlos RC. Diagnostic performance of stress cardiac magnetic resonance imaging in the detection of coronary artery disease. J Am Coll Cardiol. 2007;50:1343-53.

5. Li M, Zhou T, Yang L-F, Peng Z-H, Ding J, Sun G. Diagnostic accuracy of myocardial magnetic resonance perfusion to diagnose ischemic stenosis with fractional flow reserve as reference: systematic review and metaanalysis. JACC Cardiovasc Imaging. 2014;7:1098-105.

6. Patel AR, Antkowiak PF, Nandalur KR, West AM, Salerno M, Arora V, et al. Assessment of advanced coronary artery disease: advantages of quantitative cardiac magnetic resonance perfusion analysis. J Am Coll Cardiol. 2010;56: $561-9$.

7. Ferreira PF, Gatehouse PD, Mohiaddin RH, Firmin DN. Cardiovascular magnetic resonance artefacts. J Cardiovasc Magn Reson. 2013;15:41.

8. Chiribiri A, Leuzzi S, Conte MR, Bongioanni S, Bratis K, Olivotti L, et al. Rest perfusion abnormalities in hypertrophic cardiomyopathy: correlation with myocardial fibrosis and risk factors for sudden cardiac death. Clin Radiol. 2015;70:495-501.

9. Villa ADM, Sammut E, Zarinabad N, Carr-White G, Lee J, Bettencourt N, et al. Microvascular ischemia in hypertrophic cardiomyopathy: new insights from high-resolution combined quantification of perfusion and late gadolinium enhancement. J Cardiovasc Magn Reson. 2016;18:4.

10. Klem I, Heitner JF, Shah DJ, Sketch MH, Behar V, Weinsaft J, et al. Improved detection of coronary artery disease by stress perfusion cardiovascular magnetic resonance with the use of delayed enhancement infarction imaging. J Am Coll Cardiol. 2006:47:1630-8.
11. Schulz-Menger J, Bluemke DA, Bremerich J, Flamm SD, Fogel MA, Friedrich $M G$, et al. Standardized image interpretation and post processing in cardiovascular magnetic resonance: Society for Cardiovascular Magnetic Resonance (SCMR) Board of Trustees Task Force on Standardized Post Processing. J Cardiovasc Magn Reson. 2013;15:35 BioMed Central Ltd.

12. Plein S, Schulz-Menger J, Almeida A, Mahrholdt H, Rademakers F, Pennell D, et al. Training and accreditation in cardiovascular magnetic resonance in Europe: a position statement of the working group on cardiovascular magnetic resonance of the European Society of Cardiology. Eur Heart J. 2011;32:793-8 Oxford University Press.

13. Ishida M, Schuster A, Morton G, Chiribiri A, Hussain ST, Paul M, et al. Development of a universal dual-bolus injection scheme for the quantitative assessment of myocardial perfusion cardiovascular magnetic resonance. J Cardiovasc Magnetic Resonance. 2011;13:28.

14. Cerqueira MD, Weissman NJ, Dilsizian V, Jacobs AK, Kaul S, Laskey WK, et al. Standardized myocardial segmentation and nomenclature for tomographic imaging of the heart a statement for healthcare professionals from the cardiac imaging committee of the council on clinical cardiology of the American heart association. Int J Cardiovasc Imaging. 2002;18:539-42. Lippincott Williams \& Wilkins. Available from: http://circ.ahajournals.org/cgi/doi/10.1161/hc0402.102975.

15. Kramer CM, Barkhausen J, Flamm SD, Kim RJ, Nagel E. Standardized cardiovascular magnetic resonance (CMR) protocols 2013 update. J Cardiovasc Magnetic Resonance. 2013;15:91.

16. Kim RJ, Wu E, Rafael A, Chen E-L, Parker MA, Simonetti O, et al. The use of contrast-enhanced magnetic resonance imaging to identify reversible myocardial dysfunction. N Engl J Med. 2000;343:1445-53.

17. Hautvast G, Chiribiri A, Zarinabad N, Schuster A, Breeuwer M, Nagel E. Myocardial blood flow quantification from MRI by deconvolution using an exponential approximation basis. IEEE Trans Biomed Eng. 2012;59:2060-7.

18. Wilke N, Jerosch-Herold M, Wang Y, Huang Y, Christensen BV, Stillman AE, et al. Myocardial perfusion reserve: assessment with multisection, quantitative, first-pass MR imaging. Radiology. 1997;204:373-84.

19. Jerosch-Herold M, Wilke N, Stillman AE. Magnetic resonance quantification of the myocardial perfusion reserve with a Fermi function model for constrained deconvolution. Med Phys. 1998;25:73-84.

20. Zarinabad N, Chiribiri A, Hautvast GLTF, Ishida M, Schuster A, Cvetkovic Z, et al. Voxel-wise quantification of myocardial perfusion by cardiac magnetic resonance. Feasibility and methods comparison. Magn Reson Med. 2012;68:1994-2004.

21. Sammut E, Zarinabad N, Wesolowski R, Morton G, Chen Z, Sohal M, et al. Feasibility of high-resolution quantitative perfusion analysis in patients with heart failure. J Cardiovasc Magn Reson. 2015;17:13.

22. Lockie T, Ishida M, Perera D, Chiribiri A, De Silva K, Kozerke S, et al. Highresolution magnetic resonance myocardial perfusion imaging at 3.0-Tesla to detect hemodynamically significant coronary stenoses as determined by fractional flow reserve. J Am Coll Cardiol. 2011;57:70-5.

23. Sammut EC, Villa ADM, Di Giovine G, Dancy L, Bosio F, Gibbs T, et al. Prognostic value of quantitative stress perfusion cardiac magnetic resonance. JACC Cardiovasc Imaging. 2018;11(5):686-94. https://doi.org/10. 1016/j.jcmg.2017.07.022.

24. Fihn SD, Gardin JM, Abrams J, Berra K, Blankenship JC, Dallas AP, et al. ACCF/AHA/ACP/AATS/PCNA/SCAI/STS guideline for the diagnosis and management of patients with stable ischemic heart disease: a report of the American College of Cardiology Foundation/American Heart Association task force on practice guidelines, and the American College of Physicians, American Association for Thoracic Surgery, Preventive Cardiovascular Nurses Association, Society for Cardiovascular Angiography and Interventions, and Society of Thoracic Surgeons. Circulation. 2012;126(25):e354-471. https://doi. org/10.1161/CIR.0b013e318277d6a0.

25. American College of Cardiology Foundation Task Force on Expert Consensus Documents, Hundley WG, Bluemke DA, Finn JP, Flamm SD, Fogel MA, et al. ACCF/ACR/AHA/NASCI/SCMR expert consensus document on cardiovascular magnetic resonance: a report of the American College of Cardiology Foundation task force on expert consensus documents. J Am Coll Cardiol. 2010;55(23):2614-62. https://doi.org/10.1016/j.jacc.2009.11.011.

26. Greenwood JP, Maredia N, Younger JF, Brown JM, Nixon J, Everett CC, et al. Cardiovascular magnetic resonance and single-photon emission computed tomography for diagnosis of coronary heart disease (CE-MARC): a prospective trial. Lancet. 2012;379:453-60.

27. Schwitter J, Wacker CM, Wilke N, Al-Saadi N, Sauer E, Huettle K, et al. MR-IMPACT II: magnetic resonance imaging for myocardial perfusion assessment in coronary artery disease trial: perfusion-cardiac magnetic resonance vs. single-photon 
emission computed tomography for the detection of coronary artery disease: a comparative multicentre, multivendor trial. Eur Heart J. 2013;34:775-81.

28. Patel MR, Peterson ED, Dai D, Brennan JM, Redberg RF, Anderson HV, et al. Low diagnostic yield of elective coronary angiography. N Engl J Med. 2010; 362:886-95. Massachusetts Medical Society.

29. Kim RJ, De Roos A, Fleck E, Higgins CB, Pohost GM, Prince M, et al. Guidelines for training in Cardiovascular Magnetic Resonance (CMR). J Cardiovasc Magn Reson. 2007;9:3-4. https://doi.org/10.1080/ 10976640600778064

30. Biglands JD, Ibraheem M, Magee DR, Radjenovic A, Plein S, Greenwood JP. Quantitative myocardial perfusion imaging versus visual analysis in diagnosing myocardial ischemia: a CE-MARC substudy. JACC: Cardiovascular Imaging. 2018;11:711-8.

31. Kruger J, Dunning D. Unskilled and unaware of it: how difficulties in recognizing one's own incompetence lead to inflated self-assessments. J Pers Soc Psychol. 1999;77:1121-34.

32. Biglands JD, Magee DR, Sourbron SP, Plein S, Greenwood JP, Radjenovic A. Comparison of the diagnostic performance of four quantitative myocardial perfusion estimation methods used in cardiac MR imaging: CE-MARC substudy. Radiology. 2015;275:393-402 Radiological Society of North America.

33. Morton G, Chiribiri A, Ishida M, Hussain ST, Schuster A, Indermuehle A, et al. Quantification of Absolute Myocardial Perfusion in Patients With Coronary Artery Disease: Comparison Between Cardiovascular Magnetic Resonance and Positron Emission Tomography. J Am Coll Cardiol. 2012;60:1546-55.

34. Bernhardt $P$, Walcher T, Rottbauer W, Wöhrle J. Quantification of myocardial perfusion reserve at 1.5 and 3.0 tesla: a comparison to fractional flow reserve. Int J Cardiovasc Imaging. 2012;28:2049-56.

35. Mordini FE, Haddad T, Hsu LY, Kellman P, Lowrey TB, Aletras AH, et al. Diagnostic accuracy of stress perfusion CMR in comparison with quantitative coronary angiography: fully quantitative, semiquantitative, and qualitative assessment. JACC Cardiovasc Imaging. 2014;7:14-22.

36. Zarinabad N, Hautvast GLTF, Sammut E, Arujuna A, Breeuwer M, Nagel E, et al. Effects of tracer arrival time on the accuracy of high-resolution (voxelwise) myocardial perfusion maps from contrast-enhanced first-pass perfusion magnetic resonance. Biomedical engineering, IEEE transactions on IEEE. 2014;61:2499-506.

37. Kellman P, Hansen MS, Nielles Vallespin S, Nickander J, Themudo R, Ugander $M$, et al. Myocardial perfusion cardiovascular magnetic resonance: optimized dual sequence and reconstruction for quantification. J Cardiovasc Magn Reson. 2017;19:43.

38. Jacobs M, Benovoy M, Chang L-C, Arai AE, Hsu LY. Evaluation of an automated method for arterial input function detection for first-pass myocardial perfusion cardiovascular magnetic resonance. J Cardiovasc Magn Reson. 2016;18:17.

39. Hsu LY, Jacobs M, Benovoy M, Ta AD, Conn HM, Winkler S, et al. Diagnostic performance of fully automated pixel-wise quantitative myocardial perfusion imaging by cardiovascular magnetic resonance. JACC: Cardiovascular Imaging. 2018;11:697-707.

40. Gatehouse P, Lyne J, Smith G, Pennell D, Firmin D. T2* effects in the dualsequence method for high-dose first-pass myocardial perfusion. J Magn Reson Imaging. 2006;24:1168-71.

Ready to submit your research? Choose BMC and benefit from:

- fast, convenient online submission

- thorough peer review by experienced researchers in your field

- rapid publication on acceptance

- support for research data, including large and complex data types

- gold Open Access which fosters wider collaboration and increased citations

- maximum visibility for your research: over $100 \mathrm{M}$ website views per year

At BMC, research is always in progress.

Learn more biomedcentral.com/submissions 